\title{
Nuevas tecnologías para la interpretación y promoción de los recursos turísticos culturales
}

\author{
José L. Caro* Ana Luque** Belen Zayas*** \\ Universidad de Málaga (España)
}

\begin{abstract}
Resumen: Las Tecnologías de la Información y las Comunicaciones (NTIC) han revolucionado la forma de promocionar e interpretar el patrimonio cultural en el mundo del turismo. Actualmente cualquier destino que pretenda ser competitivo debe actualizar continuamente toda aquella información que pueda ser de interés para el visitante.

El turista cuya principal motivación es la cultura, se caracteriza por requerir gran cantidad de información y ser consumidor de TICs. Los usuarios se han transformando en usuarios 2.0, caracterizados por estar altamente conectados y por ser generadores de información y, sobre todo opinión en redes sociales, blogs, etc., siendo claves en los sistemas de reputación on-line.

En este artículo se analiza y clasifica las TIC vinculadas con la interpretación, promoción y puesta en explotación turística de recursos culturales. Así, se abordarán tanto herramientas más tradicionales como tecnologías de una actualidad manifiesta en el ámbito de la promoción y puesta en valor del patrimonio cultural. Estos instrumentos además de ser vehículo para la promoción, marketing e incluso para la planificación del destino turístico, ofrecen la oportunidad para mejorar la interpretación y gestión del patrimonio cultural en dichos espacios.
\end{abstract}

Palabras Clave: Turismo Cultural, SIG, Web 2.0, Turismo 2.0, Realidad Aumentada, 3D.

\section{New technologies for the interpretation and promotion of cultural tourism resources}

Abstract: The Information Technology and Communications (ICT) have revolutionized the way to promote and interpret the cultural heritage in the world of tourism. Currently any destination that aims to be competitive must continually update all information that may be of interest to the visitor.

The tourist whose main motivation is the culture is characterized by requiring large amount of consumer information and ICT. Users have transformed into a 2.0 users, characterized by being highly connected and be creators of information and put their opinion on all social networks, blogs, etc. All of this contributes in reputation systems on-line.

This article analyzes and classifies ICT related to the interpretation, implementation and promotion of cultural tourism resources exploitation. So, the more traditional tools such as technology news manifests in the area of the promotion and enhancement of cultural heritage will be addressed. These instruments besides being a vehicle for the promotion, marketing and even for planning tourist destination, offering the opportunity to improve the interpretation and management of cultural heritage in such spaces.

Keywords: Cultural Tourism, GIS; Web 2.0, Tourism 2.0, Augmented Reality, 3D.

\section{Introducción}

El turismo cultural se ha ido posicionando poco a poco como uno de los segmentos más desarrollados en el universo turístico mundial (Rypkema, D. et al, 2011). Los recursos de esta tipología son bienes muebles/inmuebles, paisaje e incluso patrimonio cultural intangible (música, folclore, etc.) y suponen

\footnotetext{
* Departamento Lenguajes y Ciencias de la Computación, Facultad de Turismo, Universidad de Málaga; E-mail: jlcaro@uma.es

** Departamento de Geografía, Facultad de Turismo (Universidad de Málaga; E-mail: geoana@uma.es

****mzayfer@uma.es
} 
un recurso básico para los denominados destinos "patrimoniales", o bien un recurso complementario de interés para otro tipo de destinos turísticos. El poder de atracción de un espacio turístico está directamente relacionado con la capacidad de difusión de su patrimonio, aspecto vital para el segmento del turismo cultural ya que, el turista cultural suele ser un gran consumidor de información en las fases de anticipación, experiencia y recreación del viaje turístico, además de no estar sometido a la temporalidad o a la moda (Mallor, González-Gallarza, Fayos, 2013).

Las TICs (Tecnologías de la Información y las Comunicaciones) son actualmente una herramienta vital para la difusión del patrimonio cultural ya que, por un lado, han experimentado un fuerte proceso de diversificación en los últimos años y, junto a los tradicionales canales web se han desarrollado multitud de herramientas de la web 2.0 (redes sociales); por otro lado, la socialización de la tecnología móvil, permite al gran público contar con soportes de altas prestaciones que posibilitan el uso de servicios de geolocalización, acceder a potentes bases de datos e incluso implementar sistemas de realidad aumentada (Timothy, 2011).

Toda esta serie de aspectos marcan una pauta importante, tanto en la difusión como en la reputación on-line de los destinos turísticos, creándose un marco de referencia que deber ser atendido, de ahí que este artículo plantee como objetivo fundamental la presentación de dichas tecnologías, y ofrezca un foro de discusión sobre su uso en el marco del turismo cultural. Esta pretensión demanda la caracterización del turismo cultural y su importancia cuantitativa, la definición de conceptos como el de usuario 2.0, turista 2.0 y travel 2.0 y una reflexión crítica sobre las oportunidades y riesgos que el uso de estas nuevas tecnologías plantean frente a métodos más tradicionales.

\section{Caracterización del turismo cultural}

El fuerte desarrollo experimentado por el turismo cultural en los últimos años, se enmarca en los cambios acaecidos en los destinos turísticos ante los procesos de diversificación y especialización de la demanda, que obligan a estos espacios a una búsqueda constante de singularización y diferenciación de sus productos que atiendan este consumo individualizado (Antón\&González, 2008).

Estos rasgos, propios de los espacios turísticos en la era postfordista, vienen determinados por la aparición de un nuevo tipo de turista, que valora más aquello que hace durante el viaje que el lugar en sí, caracterizándose por una búsqueda constante de nuevas experiencias básicamente en el ámbito de la naturaleza, la salud y la cultura (Antón\&González, 2008) y es, en este nuevo contexto motivacional, donde surgen multitud de turismos específicos que, por su enorme diversidad, suelen agruparse en áreas temáticas afines, entre las que la esfera cultural adquiere una gran relevancia (incluyendo segmentos específicos del tipo histórico-artístico-cultural, folclórico, gastronómico, religioso, etc.) (Torres, E., 2006).

La aparición del concepto de turismo cultural es relativamente reciente, aunque sus orígenes podrían remontarse a la antigüedad y a las experiencias viajeras transmitidas a modo de crónicas de personajes históricos tales como el emperador romano Adriano, en donde se plasma, entre otras cosas, un interés y búsqueda de conocimiento de la cultura de aquellos lugares que visita. También será representativo para el nacimiento de esta tipología turística el Grand Tour, viaje realizado por jóvenes británicos, fundamentalmente de clase alta, entre los siglos XVII y principios del siglo XIX como vehículo de formación y esparcimiento, y cuyo destino eran los lugares de referencia cultural europeos tales como Italia. Sin embargo, el hito que marcará la historia del turismo cultural tendrá lugar en 1841 gracias a Thomas Cook, el primer organizador de viajes turísticos (Faraldo \& Rodriguez, 2013).

El turismo cultural constituye una forma de turismo que obedece a necesidades y motivos propios de los consumidores por lo que su delimitación es muy subjetiva (Mallor et al, 2013). De ahí que sea un segmento complejo de definir ya que, el propio concepto de "cultura" abarca un abanico amplio de ideas (forma de vidas, monumentos, bienes muebles, etc.) que, vinculadas con la práctica turística, generan productos de muy diverso tipo, no obstante, cualquier turista cultural se va a caracterizar por el deseo que subyace en el mismo a la hora de seleccionar un destino de conocer otras culturas (sea cual sea la vertiente de la misma que le atraiga prioritariamente) (Martinez \& Herráez, 2008). En resumen, la pluralidad de turistas, la juventud del sector y la diversidad de productos relacionados, explican la complejidad de definir el segmento (McKercher y Du Cros, 2002).

Esta diversidad de motivaciones y de recursos condicionan que aparezcan tesis de diverso tipo, algunas muy generalistas, que definen el turismo cultural como "aquella forma de turismo que tiene por objeto, entre otros fines, el conocimiento de monumentos y sitios histórico - artísticos" (ICOMOS, 1976), y otras 
más específicas, como la aportada por la OMT en el año 1995 "aquella tipología turística que incluye los movimientos de las personas por motivaciones esencialmente culturales, tales como viajes de estudio, artes escénicas y visitas culturales, viajes a festivales y otros eventos culturales, visitas a sitios y monumentos, viajes para estudiar la naturaleza, el folklore o el arte, y las peregrinaciones" (OMT, 1995). Junta a estas, aparecen definiciones bastante técnicas y conceptuales, como las aportadas por Richards en el año 2001, que técnicamente lo definió como "todos los movimientos de personas hacia atracciones culturales específicas, tales como lugares patrimoniales, manifestaciones artísticas y culturales, artes y teatro, fuera de su lugar habitual de residencia", y conceptualmente como "el movimiento de personas a lugares de interés cultural lejos de su lugar de residencia habitual, con la intención de recopilar información y experiencias nuevas para satisfacer sus necesidades culturales" (Richards, 2001).

Todas las definiciones presentan un principio común, el interés por parte del turista cultural de conocer diversos elementos de la cultura, es decir, del patrimonio a la hora de seleccionar un destino. Sin embargo, también resulta difícil precisar qué es patrimonio cultural y cultura. Se entiende por patrimonio cultural "el conjunto de bienes muebles, inmuebles e inmateriales que hemos heredado del pasado y que hemos decidido que merece la pena proteger como parte de nuestras señas de identidad social e histórica" (Querol, 2010). Así, el patrimonio cultural abarca elementos físicos como museos, lugares y monumentos históricos, y sitios arqueológicos, y elementos intangibles como son las formas de vida, tradiciones, el idioma, que forman parte de la identidad de una comunidad (Hierro \& Fernández, 2013).

En la actualidad Europa es un destino clave para los turistas culturales ya que concentra recursos de enorme interés, muchos de ellos declarados patrimonio de la humanidad por la UNESCO, en concreto, España ocupa el segundo puesto respecto al número de bienes declarados Patrimonio de la Humanidad en el continente europeo después de Italia (UNESCO, 2014). La calidad de los recursos, motiva que España se esté convirtiendo en un destino de turismo cultural referente a escala internacional de hecho, España recibió en el año 2013 un total de 60.6 millones de turistas, convirtiéndose en el tercer país receptor a escala mundial (Romero \& Delgado, 2014), de los cuales, un total de 11,7 millones de viajes se realizaron por motivaciones relacionadas con el ámbito de la cultura, inclusive, estas cifras podrían incrementarse si se atiende a un concepto de cultura amplio que incluya motivaciones del tipo gastronómico, enológico o paisajístico (IAB, 2012).

En otro orden de cosas, si con respecto a la definición no existía un claro consenso, tampoco existe en lo relativo a su tipificación, motivo por el cual es un tema en continua revisión (Stylianou-Lambert, 2010). Una de las principales fuentes de discrepancia entre los estudios afecta a temas tan ajenos como el ocio e incluso el deporte, ya que el concepto de ocio no es un componente del turismo cultural para la WTO (2005), mientras que autores como Richards (2001) lo considera como un elemento necesario. Por su parte, hay autores como Cluzeau (2000) que no admiten el deporte como forma de turismo cultural, sin embargo, en Barcelona el segmento del turismo cultural había incrementado su importancia entre 1994 y 2002 debido a la atracción ejercida por el museo del FC Barcelona, tal como recoge Mallor et al (2013).

Como los restantes turismos específicos, el turismo cultural posee necesidades complejas con respecto a la conformación de la oferta, desde diversos puntos de vista: suelen demandar procesos más complejos con respecto a la organización de las actividades, con respecto a la cualificación de recursos, se encuentran muy afectados por los avances tecnológicos, demandan importantes inversiones, etc. Además de esto, suelen ser tipologías turísticas que poseen rasgos específicos desde el punto de vista de la demanda, por ejemplo, tener un gasto medio superior a los usuarios de turismos genéricos, altos niveles de exigencia centrados en aspectos propios de la motivación, diversidad de subtipologías en relación con los intereses específicos, etc. A principio de los años noventa, Ashworth y Turnbridge (1990) identificaron dos tipologías de turista cultural: el de intención (cuando el visitante se muestra interesado por los diversos atractivos patrimoniales de un destino) y el incidental (cuando el turista no siente una motivación primaria hacia los recursos patrimoniales). Por su parte, Mackercher y Du Cros en el año 2002 ampliaron esta división a cinco tipos de turista cultural en función de diversos aspectos motivacionales (Mackercher\&Du Cros, 2002):

- Turista cultural completo: cuyo motivo principal es la cultura y es lo que le mueve al destino buscando una experiencia cultural en profundidad.

- Turista cultural visitante: su motivo principal es el turismo cultural para visitar el sitio pero busca una experiencia menos profunda.

- Turista cultural descubridor: es un turista que no viaja por motivos culturales pero que acaba teniendo una experiencia cultural, algunas veces profunda. 
- Turista cultural casual: es el turista que no tiene como motivo principal las actividades culturales, pero que incluye alguna actividad cultural del destino en su viaje.

- Turista cultural accidental: es el turista que no tiene como motivo principal el motivo cultural pero que accidentalmente participa en alguna actividad.

En cualquier caso, existen dos rasgos característicos en los turistas culturales, tal como recogen diversos estudios (ATLAS, 1997; Richards, 2001), poseer un alto poder adquisitivo y un alto nivel académico.

En España, el denominado "turista cultural" se caracteriza por ser un turista de edad media, entre 25 y 44 años, sin diferencia significativa entre sexos y que habitualmente demanda estancias cortas (superando a las tradicionales vacaciones de verano). Con respecto a la forma de organizar el viaje, suele ser en bastantes ocasiones por cuenta ajena (sobre todo en el destino España $>80 \%$ ) y cuyo alojamiento favorito es el hotel (88,2\%). Junto a esto, suele ser un turista que demanda gran cantidad de información, recurriendo a Internet como fuente básica.

\section{Herramientas para la promoción e interpretación del patrimonio en destinos turísticos culturales}

\subsection{Nuevas Tecnologías de la Información y la Comunicación (NTIC) y turismo}

Desde hace más de una década el uso de nuevas tecnologías se ha convertido de manera generalizada en algo indispensable tanto en la creación y gestión de un destino turístico como en la realización del viaje. Esta importancia ha ido creciendo, desde los primeros sistemas de información para las reservas de vuelos, a partir de los cuales se generaron los modernos GDS (Sistemas Globales de Distribución), hasta los actuales sistemas de información que copan el ámbito de la gestión. Dentro de esta categoría podemos destacar un abanico de tecnologías: bases de datos, redes de datos, ingeniería del software, sistemas de información geográfica, modelado 3D, sistemas de posicionamiento y un largo etc., que pueden trabajar de forma individual o conjunta para labores de gestión, de desarrollo, de auditoría y de promoción para los destinos (Poon, 1993; Sheldon, 1997; Inkpen,G., 1998; O'Connor, 1999). Estos instrumentos han supuesto un avance y evolución tecnológica en el ámbito turístico, generándose una disciplina que abarca los desarrollos tecnológicos para el turismo bajo el término eTourism (Buhalis \& Law; 2008; Buhalis, 2012).

Hay multitud de herramientas en el marco de las NTIC vinculadas con la actividad turística, no obstante, por su repercusión y difusión cobra especial interés la web que, a día de hoy, se ha consolidado como clave en cualquier proceso relacionado con el turismo (promoción, difusión, venta, información, etc.) (Buhalis, 2012). Este interés se entronca en el propio proceso de evolución y mejora de la herramienta ya que, en sus inicios, la web 1.0 incluía páginas con un buen diseño, con contenidos realizados por los autores de las páginas, una interacción baja en un sentido unidireccional (usuario como lector de la información) y con un contenido fijo pero, con la incorporación de las bases de datos, se fueron dinamizando.

La web 2.0 cambia radicalmente este panorama. Los tradicionales sitios web se transforman en aplicaciones web, se generan noticias, se comparte información entre sitios, aparecen las llamadas aplicaciones 2.0 y, sobre todo, comienza a generarse contenidos de una forma más descentralizada. El usuario pasa de ser un mero lector a ser un lector/escritor convirtiéndose en lo que llamamos usuario 2.0 (figura 2). Este usuario ha desarrollado un conjunto de nuevas capacidades, habilidades y costumbres relacionadas con su interacción con las nuevas herramientas de la web 2.0 (foros, blogs, redes sociales, buscadores avanzados, sistemas de recomendación, reputación on-line, etc.) las cuales generan nuevas demandas de información.

La Web 2.0, más que un conjunto de herramientas, supone un cambio radical en la forma del acceso a la información (figura 1) y supone un valor añadido a la misma. En términos más concretos es un avance en aspectos tan importantes como la interactividad y la colaboración, así como en la generación de contenidos on-line (Liburd, 2012). 
Fig. 1: Evolución de la web 1.0 a web 2.0 (Fuente: elaboración propia)

\begin{tabular}{|c|c|c|}
\hline Usuario Lector & Web 2.0 & $\begin{array}{c}\text { Usuario Productor } \\
\text { información }\end{array}$ \\
\hline 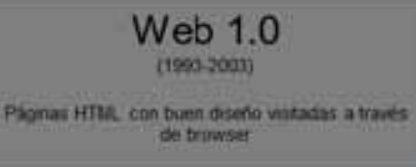 & & 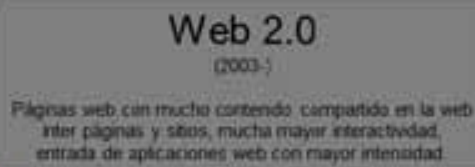 \\
\hline "Lectura" & Modo & "Escribir" \& Contribuir \\
\hline "Página" & $\begin{array}{l}\text { Unidad Primaria de } \\
\text { información }\end{array}$ & "Post / Registro" \\
\hline "Estático" & Estado de las páginas & "Dinámico" \\
\hline Navegador web & $\begin{array}{l}\text { Se lee la información a } \\
\text { traves de... }\end{array}$ & $\begin{array}{l}\text { Navegadores, RSS, cualquier } \\
\text { sistema }\end{array}$ \\
\hline "Cliente-servidor" & Arquitectura & $\begin{array}{c}\text { "Web Services" } \\
\text { (es cliente /servidor) }\end{array}$ \\
\hline Web Coders & Contenido creado por ... & Cualquiera \\
\hline "geeks" & Dominio de... & "mass amatuerization" \\
\hline
\end{tabular}

Fig. 2: El usuario 2.0

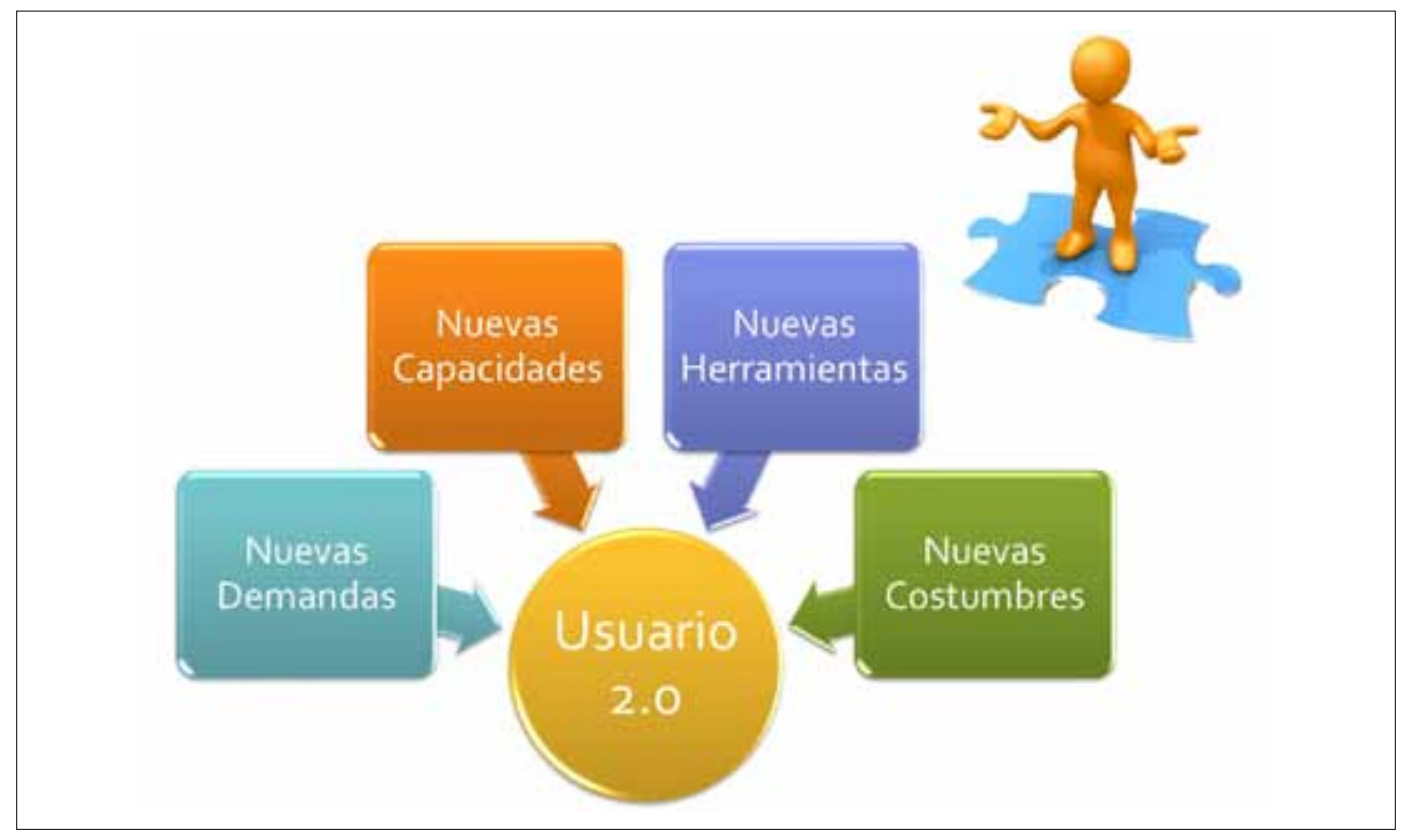

Fuente: elaboración propia 
Por otro lado, los profesionales del turismo utilizan estas herramientas para, por ejemplo, reconducir la forma en la que se realiza la promoción, abriendo sus miras a las web tradicionales, CMS (gestores de contenidos) y a las herramientas de la ya referida web 2.0., en el proceso de venta y promoción vía internet en el círculo [crear deseo, búsqueda de un producto, comparar ofertas, reservar/comprar, viajar, visitar/alojar, hablar del tema] (Cybermassif, 2010) (ver figura 3). En concreto, en el segmento del turismo cultural, la difusión y promoción a través de la web, es un tema bastante desarrollado desde hace años, fundamentalmente, en los museos, tanto por los rasgos que definen a esta tipología de turista como por los bienes que suponen los recursos que lo singularizan (Mokre, 1998; Scali et. al, 2002 ; Syliani et al. 2009).

\section{Figura 3: Ciclo de compra (a partir de Cybermassif, 2010)}

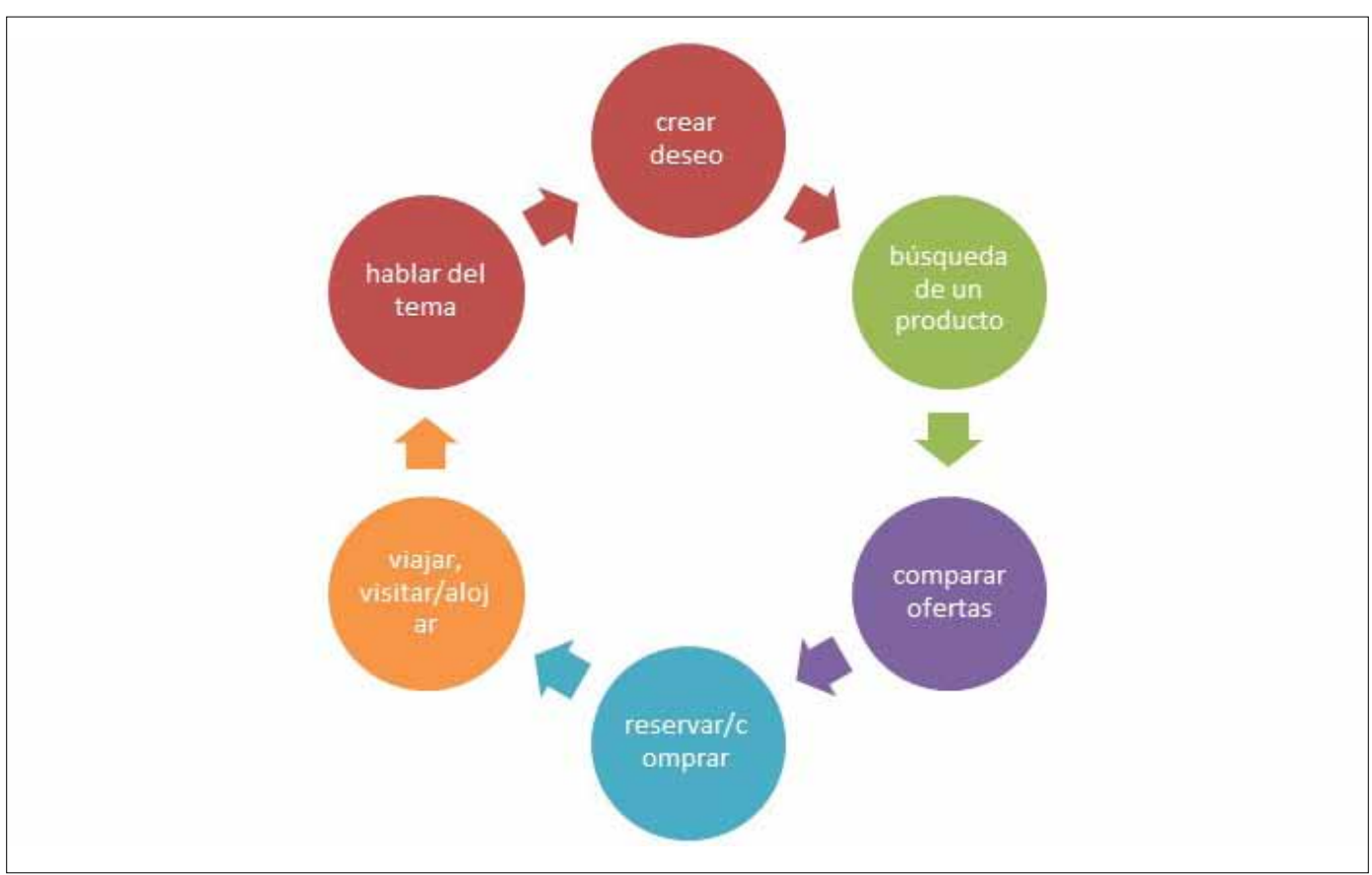

El usuario 2.0, cuando nos referimos al ámbito del turismo, se transforma en el turista 2.0. El viajero lleva utilizando la web desde hace muchos años para localizar información, sin embargo, en la actualidad se ha convertido en el medio informativo preferente, utilizándolo el $42 \%$ de los turistas: las páginas de redes sociales suponen un $13 \%$, revistas on-line $12 \%$, blogs $18 \%$, secciones de suplementos de viaje de portales de Internet con un $18 \%$, etc.; además de tener cada vez más importancia en el proceso de compra (sobre el 26\%) tanto para el alojamiento como para transporte (IAB, 2012). Dentro de las diversas aplicaciones utilizadas, destacan los sistemas de recomendación y reputación, es decir la consulta de información de otros usuarios resulta clave en el comportamiento a la hora de viajar. Un $35 \%$ (recomendación) y un 24\% (reputación) de los encuestados puntuaron, en la encuesta de IAB, estos aspectos con una valoración de 9-10 (IAB, 2012).

Este nuevo usuario se traduce en el concepto de travel 2.0, es decir en el uso de herramientas de la web 2.0 como blogs, redes sociales, sistemas de recomendación, integración de contenidos mediante mashups, audio, video, planificadores de viaje, etc. Pero el concepto en la actualidad abarca mucho más ya que, no supone tan sólo el uso de las herramientas sino, fundamentalmente, un cambio en su uso: el usuario utiliza las mismas en el proceso de anticipación, experiencia y recreación del viaje turístico, a lo que habría que unir un cambio en la filosofía global en el uso de la información que Philipe Wolf en 2006 (Almeida et al, 2008), Director General de PhoCusWright Inc., describió como principios básicos: 
- Colaboración desinteresada.

- Transparencia y equidad en datos, precios y contenidos. Intentando que la información sea "coherente" entre todas las fuentes.

- Aparición de redes sociales especializadas las cuales hoy en día son una herramienta importante a la hora de toma de decisiones en el ámbito del turismo (reputación on-line).

- Aumento de la velocidad y volumen en el intercambio.

- Mejora en la credibilidad de la información ya que procede de los propios viajeros.

Así, nace el concepto de ADPROSUMER (AD - anuncio, PRO - productor y SUMER - consumidor) que hace las funciones simultáneas de comprador, consumidor y recomendador de productos. Es en este aspecto en el que estas nuevas tecnologías ofrecen un cambio en el paradigma del viajero. Antes, porque busca opiniones y compra; durante porque comunica sus impresiones a través de las redes sociales, blogs y sistemas de mensajería, y, después, porque genera opiniones y reputación en el destino. Así, el traveler 2.0 usa las herramientas disponibles en todas las fases del viaje.

En el ámbito del turismo cultural, como no podía ser menos, se cumplen estas premisas. De hecho, en la primera fase (anticipación), son los tipos de turistas que más información requieren, de ahí que cobren especial interés las herramientas informativas, junto a ellas y, ya en la fase experiencial, resultan capitales aquellas que se centran en la difusión y gestión de los recursos y, por último, en la fase de recreación, las herramientas comunicativas.

\subsection{Herramientas para la difusión del patrimonio en destinos turísticos culturales}

En los apartados anteriores se ha analizado la forma en la que los turistas pertenecientes al segmento cultural se comportan y acceden a la información y el interés que, en el proceso experiencial del viaje, cobran los aspectos relacionados con la difusión de los recursos. Turismo, cultura y NTIC suponen una trilogía de enorme actualidad que abarca parcelas diversas, sin embargo, si se examinan los tópicos de la "Conference on Cultural Heritage and New Techologies" (CHNT) (principal congreso en el que se incluyen los últimos avances en TICs y patrimonio cultural) aparece que, en sus últimas ediciones, los tópicos que abordados se centran básicamente en cuatro áreas:

- Gestión: gestión del patrimonio cultural y las aplicaciones informáticas asociadas.

- Web: aplicaciones web para la difusión y registro del patrimonio (incluyéndose las redes sociales).

- GIS: abarcándose diversos aspectos como análisis geoespacial, registro espacial, etc.

- 3D: incluyen temas relacionados con la digitalización, realidad virtual, realidad aumentada, etc.

- Pero, llegados a este punto, se debe reflexionar sobre cuándo y por qué se deben emplear estas tecnologías para que, dicha reflexión, sirva de referente sobre cómo abordar su uso en el marco específico del turismo cultural, en este sentido, el empleo de estas herramientas es importante:

- Antes del viaje (fase de anticipación): porque el turista que posee más requerimiento de información es el cultural, porque se deben promocionar en la red los contenidos desarrollando la potencialidad de los mismos y, sobre todo, porque es una ventana de atracción al turista al destino.

- Durante el viaje (fase experiencial): porque la información previa se debe enriquecer, porque en la actualidad el aspecto visual puede complementar el elemento emocional, porque se desea transmitir durante la estancia del turista.

- Después (fase de recreación): porque el turista puede requerir más información sobre el destino y complementar lo visitado, porque deseamos que el turista cultural actúe en los sistemas de reputación on-line para mejorar la visibilidad del destino y nos recomiende y, porque, en definitiva, se busca su fidelización y atraer más visitantes.

El uso de TICs para difusión y revalorización de los destinos culturales ayudarán a conseguir estos objetivos. En los siguientes apartados se desarrollarán dichos aspectos.

\section{a. Redes sociales}

El uso de las redes sociales en el turismo es un tema estudiado ampliamente en la literatura científica turística (Zeng \& Gerritsen, 2014) pero es un elemento en el que aparece la dificultad inherente de conocer el impacto real en cifras para el turismo. Las redes sociales son un lugar de encuentro y una oportunidad de llegar a millones de usuarios (figura 4). La existencia de amigos 2.0 que exponen la experiencia de viaje, fotos, etc (Buhalis \& Law, 2008; Volo, 2010) son un elemento de promoción directa eWOM (Word-of-mouth) (Serra \& Salvi, 2014) que debe ser cuantificado. 


\section{Redes Sociales}

\section{Oportunidades / Integración}

- Canales con millones de usuarios

- Facebook (800 millones)

- Twitter(500 millones)

- Google + (100 millones)

- Temáticas

- YouTube (490 millones)

- MySpace (65 millones)

- Linkedla(135 millones)

- FourSquare (15 millones)

- Flickr (6o millones)

- Tripadvisor (45 millones)
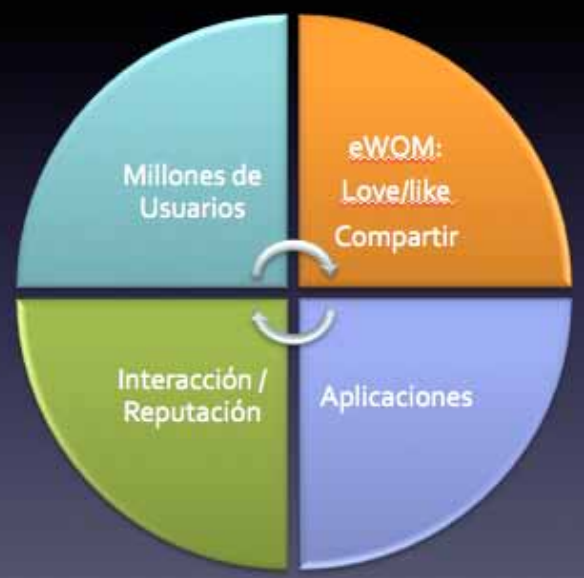

Fuente: elaboración propia

La presencia de empresas turísticas en las redes sociales es muy alta, por ejemplo, un 85,73\% de las mismas poseen página en Facebook (tabla 1) pero, de éstas el 42,81\% tiene más de una página, lo que puede ser una práctica no deseable ya que diversificaría esfuerzos entre sus seguidores (Hey, 2012). Por tanto, sería importante analizar qué uso se está haciendo de las redes sociales para maximizar su impacto en el turista.

Tabla 1: Participación de empresas turísticas en redes sociales

\begin{tabular}{|c|c|}
\hline Red Social & \% de empresas turísticas \\
\hline Facebook & 85.73 \\
\hline Twitter & 74.55 \\
\hline Flickr & 61.59 \\
\hline Youtube & 73.60 \\
\hline Foursquare & 28.89 \\
\hline Linkedin & 63.73 \\
\hline Wordpress & 28.66 \\
\hline Vimeo & 5.94 \\
\hline
\end{tabular}

Fuente: elaboración propia a partir de (Hey, 2012) 
Existen diversas aproximaciones al uso de las redes sociales en este ámbito. Éstas abarcan desde la exposición de una información general que se asimilaría a la utilización como web en la que los seguidores hacen de suscritores, hasta una forma más activa, de acuerdo al uso para el que fueron diseñadas, que incluiría un abanico de información exposición de eventos, noticias, visitas o exposiciones (Hey, 2012).

\section{b. Sistemas de Geolocalización, DTM y GIS}

Los servicios de geolocalización son el segundo gran tópico que encontramos en la puesta en valor del turismo y en concreto del turismo cultural. Existe una gran cantidad de ejemplos que hoy día son ya cotidianos para el turista. Son conocidas las soluciones de mapas como la de Google con su producto GoogleEarth o su integración en la web con Google Maps. Además, mediante la experiencia MapsGL orientada a la generación de modelos 3D de edificios singulares de interés se pueden visualizar en 3D. En el terreno de los dispositivos móviles tanto Android, mediante Google Maps, como iOS, con los mapas de Apple, hacen que estas aplicaciones sean de uso cotidiano para el turista. Antes del viaje, porque localiza los POI (Point Of Interest) sobre una cartografía y, durante el mismo, puesto que se usan para llegar al lugar o incluso emitir opiniones sobre los mismos en redes sociales como Foursquare o en sistemas de reputación on-line como Tripadvisor (tanto durante como a la finalización del viaje).

Describiendo un conjunto de iniciativas nos aproximaremos a la funcionalidad puesto que si bien los GIS desde el punto de vista de la gestión del patrimonio cultural son un elemento básico y necesario, de cara a la promoción turística se puede emplear de forma efectiva. Por ejemplo, ArqueoTur (http:// www.arqueotur.org) aúna una gran base de datos con información de yacimiento arqueológicos con un sistema cartográfico básico (usando la API de Google). Ofrece la posibilidad de marcar sobre un mapa los diferentes POI a visitar e incluso se puede extraer la información de cómo llegar para un GPS.

El modelado del terreno y generación de rutas conjugado con DTM (modelos digitales del terreno) y $3 \mathrm{D}$ ofrece la posibilidad de generar rutas navegables. Un ejemplo singular es el Camino de Santiago 2.0 - Camino Francés (Instituto Geografico Nacional, 2011). Mediante su aplicación es posible realizar una visita virtual en el camino. Ésta se nutre de un modelo digital del terreno enriquecido con puntos de interés turísticos, recursos, alojamiento y restauración para el peregrino y, sobre todo, los senderos. Sistemas como LiDAR también pueden ser empleados para la generación de modelos digitales para la preservación y difusión del patrimonio cultural, ejemplo de su uso lo encontramos en la colina de Tara (Irlanda) (Corns \& Shaw, 2009) o en Stonhenge (Bewley et al., 2005).

Uno de los aspectos que en los últimos años necesariamente ha estado de actualidad es el uso de las tecnologías móviles. La existencia de dispositivos con grandes capacidades gráficas, en lo referente a resolución y procesamiento 3D unido a la alta resolución de sus pantallas, y la incorporación de GPS y giroscopios ha abierto un mundo (que hace años existía) de enorme de posibilidades. Actualmente se pueden incorporar aplicaciones sobre cartografía on-line prácticamente en cualquier dispositivo móvil. Quizás el ejemplo que nos pueda resultar más adecuado para enlazar con la siguiente sección sea Layar una aplicación y API que permite, usando técnicas de realidad aumentada, mostrar capas de recursos. Su API se está constituyendo casi en un estándar en su categoría.

\section{c. $3 \mathrm{D}, \mathrm{VR}$ y AR}

Cómo se ha avanzado en el apartado anterior, la aplicación de tecnologías 3D es un tema recurrente que se lleva estudiando hace ya décadas. No obstante, en la actualidad es cuando realmente se está en disposición de llevarla a práctica para el turista. La aparición de smartphones, tablets y phablets con alta conectividad y gran capacidad de procesamiento gráfico hahecho posible su uso en este campo.

La tecnología 3D es un marco de referencia para lo que se conoce como aplicaciones de realidad virtual (VR) (Guttentag, 2010) y realidad aumentada (AR). En el ámbito del patrimonio se ha recurrido en incontables ocasiones a la digitalización del mismo para su difusión mediante técnicas de "dibujo". (modelado tradicional). Éste, para la difusión turística, es interesante pero carece de una característica esencial: el turista cultural va buscando la experiencia además del conocimiento y, por tanto, se deben plantear modelos más reales y, en el caso del modelado tradicional, se proporciona una idealización.

Para conseguir modelos más realistas (que puedan sustituir a la fotografía) en el proceso de digitalización del patrimonio cultural, tanto para bienes muebles como bienes inmuebles, se debe recurrir a otro tipo de técnicas. Se dispone de múltiples opciones que abarcan desde los LiDAR anteriormente mencionados, la tecnología LASER tipo faro con estaciones topográficas que permiten la digitalización de bienes inmuebles o la fotogrametría (Caro, 2012). Cada uno de estos elementos tiene ventajas e inconvenientes que se discutirán en la siguiente sección, pero todos tienen como objetivo producir modelos $3 \mathrm{D}$ de forma precisa. 
Los resultados de estas técnicas pueden ser usados para sistemas de realidad virtual, es decir sistemas que posean una alta inmersión que transporten al turista al lugar y, sobre todo, en sistemas de realidad aumentada. Los sistemas de realidad aumentada sean quizás las aplicaciones con más futuro dentro de la difusión del patrimonio porque poseen las siguientes características:

- Se aprecia la realidad, es decir el recurso cultural tal como es. La realidad aumentada aumenta una base real (imágenes del mundo real) complementada con información, bien sea textual o multimedia usando modelos 3D.

- Puede complementar la información antes de la llegada del turista al recurso (sin ofrecer información plenamente sintética).

- Se puede complementar información en el propio destino, aumentado la información visual en la propia visita al bien de interés cultural.

- Así, esta tecnología a la que se incorporan tecnologías de geoposicionamiento, 3D, computación ubicua con alta conectividad a Internet ofrece un valor añadido a la experiencia del turista 2.0 de la mano de dispositivos móviles.

\section{Conflictos y oportunidades del binomio Turismo Cultural \& NTIC}

Todas las tecnologías revisadas hasta ahora sirven de una forma efectiva para la promoción de recursos/destinos de interés cultural pero se debe reflexionar cuándo, con qué objetivo usarlas y su efectividad, siendo esta última pretensión la más compleja ya que resulta difícil medir el impacto de las mismas sobre el turista cultural, y como éstas pueden asegurar un retorno de la inversión realizada.

En el caso de las redes sociales, hay que analizar el uso que se hace de ellas y su penetración entre los seguidores. Una red social sin actividad resta turistas potenciales, de hecho hoy en día es considerado como un aspecto negativo. En el mundo de las redes sociales se debe analizar la frecuencia de actualización, el mantenimiento, las herramientas que se usan como apoyo y un largo etc., labor de community managers (Bacon, 2012). Por ejemplo, en el ámbito del turismo (Hey, 2012) encontramos que, de media, se generan 0,88 posts por semana en Facebook y 2,11 en twitter por semana. Es decir, tenemos una escasa incidencia en la actividad de la red social.

Pero no es este el único problema, existe el peligro del uso que se hace de la misma. Se puede utilizar solamente para incluir información, recibir quejas, realizar concursos y promociones, y un largo etc., sin que nada de ella esté estandarizado. Cómo sacar provecho de las redes sociales en el ámbito de la promoción turística es todavía un tema abierto. A esto habría que unir la respuesta y el grado de penetración de las mismas. El 12\% de las empresas interactúa con sus partners a través de redes sociales pero ¿̇cuál es el grado de penetración real de una campaña? ¿cómo afectan las opiniones de los usuarios? Por ejemplo, en Facebook el 56,8\% de los usuarios no responden y en Twitter el $65 \%$ no retweetean en el ámbito del turismo (para empresas con más de 50 seguidores) (Hey, 2012). Por tanto, existen aún muchos temas pendientes en las redes sociales que afectan a la forma de uso y a la métrica de su rentabilidad.

En lo que se refiere a la generación de modelos 3D, a priori se adecúa bastante a las necesidades del turista cultural. Por un lado, las herramientas de dibujo tradicionales se quedan atrás a la hora de representar la realidad, son modelos 3D diseñados. Las tecnologías LASER son muy precisas pero en muchas ocasiones la generación de texturas para representar la información se antoja escasamente realista y su coste es altamente elevado. La tecnología fotogramétrica para la generación de modelos 3D de elementos del patrimonio parece la más adecuada puesto que con un reducido coste se pueden generar modelos $3 \mathrm{D}$ precisos, con una textura absolutamente realista al generarse a partir de tomas de pares fotográficos.

Todas estas formas de digitalizar los bienes culturales para su difusión pueden ser completadas con información $3 \mathrm{D}$, textual y multimedia adicional generando sistemas de realidad aumentada que pueden ser ejecutados en dispositivos móviles. A pesar de todo esto se corre el peligro de olvidar que el turista cultural como cualquier turista quiere ver la versión original del bien y busca experiencias en el viaje. Por tanto, siempre hay que tener presente que no son un sustituto a la experiencia, sino un complemento a la misma (Guttentag, 2010).

No menos importante resultan aquellas tecnologías que se tildan como obsoletas pero que, utilizadas con imaginación, hacen crear experiencias interesantes. El uso de tecnologías mixtas (Fino et. al. 2013), repensar las existentes y, sobre todo, plantear usos novedosos de las mismas, pueden llevar a la obtención de resultados útiles para el turista. Por ejemplo, los formatos U3D y X3D en la representación de modelos en la web y en folletos digitales en PDF tradicionales (figura 5), pueden proporcionan un valor añadido sin recurrir a tecnologías caras. En éstos puede perfeccionar la imagen incrustando verdaderos modelos 3D generados a partir de la fotografía, que proporcionan un realismo exacto y que ofrecen a al turista una perspectiva nueva aumentando y mejorando la información que se presenta (Caro, 2012). 
Figura 5: Proceso de trabajo para fotogrametría y modelado 3D
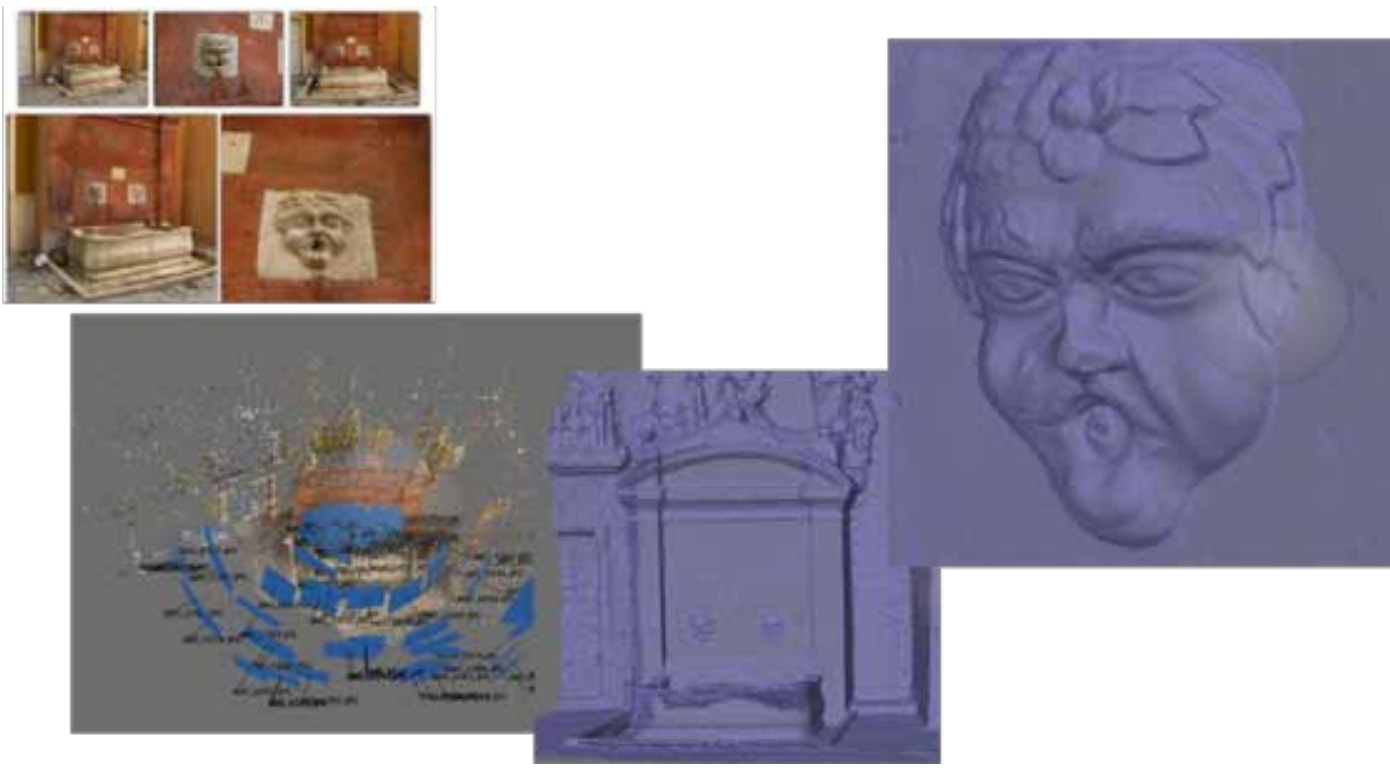

Fuente de Calle Cristo (Málaga). Elaboración propia.

Otro interesante caso, es el uso de códigos de barra o códigos QR que encontramos en NaturePlus del Natural History Museum (Londres), que consiste en una tarjeta de cartón con un código de barras incorporado que dotan al usuario de una experiencia nueva en su visita al museo. Es un sistema simple, tras darse de alta el usuario en la web del museo y, a medida que se va pasando el código de barras por cada uno de los elementos de interés, se va almacenando (en un sitio web) información adicional de los recursos seleccionados. Pero, detrás de todo esto, se esconde un sistema CRM que es capaz de registrar el perfil de los visitantes a esta sección del museo de historia natural (figura 6).

\section{Figura 6: Tarjeta NaturePlus (Natural History Museum - Londres)}

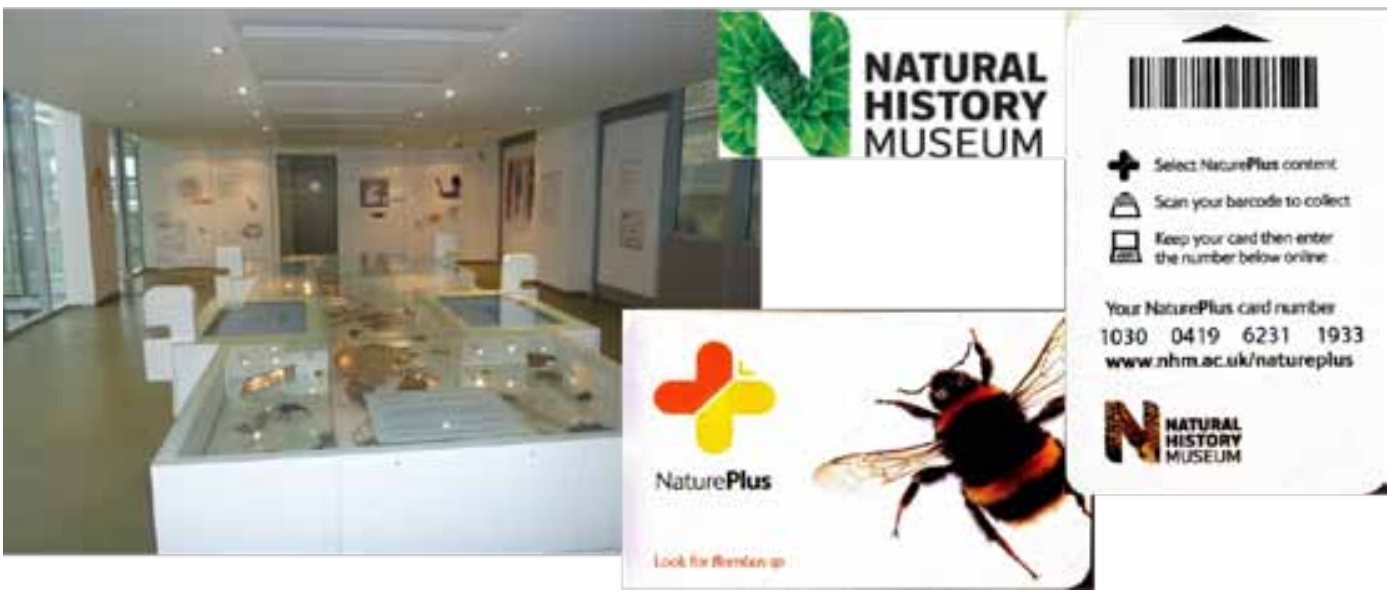

Fuente: http://www.nhm.ac.uk/ 
Con estas leves pinceladas se demuestra que no sólo se garantiza un retorno de inversión o nueva experiencias con tecnologías de alto coste, sino que además las nuevas formas de emplear la tecnología están hoy en día al alcance real de los turistas culturales. Para el destino, no sólo le garantiza un retorno de inversión, sino que se conforman como herramientas clave para promocionar bienes de interés cultural, o para conocer el perfil del visitante, a la par muy útiles para alcanzar la excelencia de los destinos turísticos culturales.

\section{Conclusiones}

A través de este trabajo se ha podido definir y repasar en cifras la importancia creciente del segmento del turismo cultural y los vínculos del mismo con las nuevas tecnologías aplicadas al sector turístico. Estas NTIC se han venido conformando en herramientas clave en la configuración de los Smart Tourism Destination, entendidos estos como "destinos turísticos innovadores, consolidados sobre una infraestructura tecnológica de vanguardia, que garantizan el desarrollo sostenible del territorio turístico, accesible para todos, que facilitan la interacción e integración del visitante con el entorno e incrementan la calidad de su experiencia en el destino" (Segittur, 2013).

Los rasgos que definen a un "destino turístico inteligente", imponen el desarrollo de un espacio turístico innovador desde un punto de vista territorial y turístico, y todo ello en el marco de una tecnología de vanguardia y promoviendo una universalización del sistema desde el punto de vista de la accesibilidad al mismo. Por tanto, es un nuevo concepto de destino turístico que plantea una dimensión más dinámica de la actividad donde los múltiples agentes que la conforman están conectados por un flujo constante de información, produciendo productos y servicios diversos (Segittur, 2013; Lamsfus \& Alzur-Sorzabal, 2013).

Los destinos turísticos culturales se enfrentan a los retos impuestos por esta nueva concepción de "destino turístico" donde el desarrollo de una infraestructura tecnológica de vanguardia adquiere un peso capital, ya que la misma debe garantizar la eco-eficiencia del destino, mejorar la conexión entre destinos, personas y servicios, crear conexiones emocionales entre el visitante y el destino, etc., y, para en el caso de los destinos culturales, facilitar y mejorar la interpretación del patrimonio. Este es un campo donde se abren enormes posibilidades para el segmento del turismo cultural, por ejemplo, desarrollo de video-guías, de dispositivos ópticos inteligentes que faciliten los procesos de inmersión histórica, experiencias personalizadas en los museos, técnicas de videomapping y holografía, etc., y otras ya referenciadas como las técnicas de realidad aumentada.

No obstante, las posibilidades que abren estas herramientas para el turismo cultural en campos como la difusión, comercialización, promoción, etc., no deben convertir a los destinos en esclavos de la tecnología, de hecho, cualquier inversión que se prevea hacer debe contar con un buen estudio de costes y de retorno de inversión ya que, en multitud de ocasiones, existen posibilidades imaginativas que aúnan tecnologías supuestamente obsoletas con nuevas tecnologías, que pueden otorgar un valor añadido y una nueva experiencia al turista, a la par que recabar información de interés para los gestores, y todo esto con una inversión mínima.

\section{Bibliografía}

Almeida, Mar Alonso, Figueroa Domecq, Cristina, Rodriguez Antón, José Miguel, Talón Ballestero 2008. "El impacto de la tecnología social en las decisiones de consumo turístico". En Guevara et al. (Eds).

VII Congreso Turismo y Tecnologías de la Información y las Comunicaciones (TuriTec2008). Málaga. Anton, S. \& González, F. (coord.)

2008. A propósito del turismo. La construcción social del espacio. Barcelona: UOC.

Ashworth, G. \& Turnbridge, J.

1990. The tourist-historic city, Londres: Belhaven

ATLAS

1997. ATLAS European Project en http://www.atlasproject.eu/ accedido 2 junio 2014.

Bacon, Jono

2012. The art of community manager. Ed. O'Reilly. 
Bewley, R. H. ; Crutchley, S. ; Shell, C.A.

2005. New light on an ancient landscape: LIDAR survey in the Stonehenge World Heritage Site. Antiquity, 79 (305): 636-647.

Buahalis, D.

2012. eTourism. Information Technology for Strategic Tourism Management. Ed. Prentice Hall

Buhalis, D., \& Law, R.

2008. "Progress in information technology and tourism management: 20 years on and 10 years after the Internet-The state of eTourism research.” Tourism Management, 29(4):609-623.

Caro, J.L.

2012. "Fotogrametría y modelado 3D: un caso práctico para la difusión del patrimonio y su promoción turística". En TuriTec2012. Congreso Turismo y Tecnologías de la Información y las Comunicaciones. Málaga: Universidad de Málaga.

Cho, V.

1998. "World Wide Web resources". Annals of Tourism Research, 25(2): 518-521.

Cluzeau, C.O.

2000. Le tourisme culturel $2^{\mathrm{a}}$ ed., París : Presses Universitaires de France-PUF.

Corns, A., \& Shaw, R.

2009. "High resolution 3-dimensional documentation of archaeological monuments \& landscapes using airborne LiDAR". Journal of Cultural Heritage, 10:72-77.

Cuccia T., Cellini R.

2007. "Is cultural heritage really important for tourists? A contingent rating study". Applied Economy 39(2):261-271

Cybermassif

2010. "Guía práctica de las TICs en el sector del Turismo". Acceso Marzo 2010 en http://www.cybersudoe. eu/es/uploads/guides_es/Guia_cybersudoe_TIC_aplicadas_en_el_sector_del_tourismo_es.pdf accedido 10 marzo 2014. Proyecto SUDOE. Interreg IVB

Faraldo, Jose M.; Rodríguez-López, Carolina.

2013. Introducción a la Historia del Turismo. Ed. Alianza Editorial.

Fino, E. R., Martín-Gutiérrez, J., Fernández, M. D. M., \& Davara, E. A.

2013. "Interactive Tourist Guide: Connecting Web 2.0, Augmented Reality and QR Codes". Procedia Computer Science. 25: 338-344.

Guttentag, D. A.

2010. "Virtual reality: Applications and implications for tourism". Tourism Management, 31(5): 637-651

Hey Avenue

2012. "El uso de las redes sociales en el sector turístico: Análisis cualitativo de estrategias de comunicación y Social Media marketing" en FITUR 2012 http://heyav.com/smsharing/UsoRedesTurismoHeyAV. pdf accesed 20/03/2014

Hierro, J. Al., \& Martín Fernández, J.

2013. "Activos culturales y desarrollo sostenible : la importancia económica del Patrimonio Cultural". Política y Sociedad: 1133-1147.

IAB Spain

2012. "Usos, Actitudes y Tendencias del consumidor digital en la compra y consumo de viajes. Observatorio Digital IAB Spain" en http://www.segittur.es/opencms/export/sites/segitur/.content/galerias/descargas/ documentos/Hot_Topic_Viajes_IAB_abril_20122.pdf accedido el 15/03/2014

ICOMOS

1976. International Cultural Tourism Charter. Ed. ICOMOS International Scientific Committee on Cultural Tourism

Inkpen,G.

1998. Information technology for travel and tourism. London: Addison-Wesley-Longman.

Lamsfus, C. \& Alzur-Sorzabal, A.

2013. "Theoretical framework for a tourism internet of things: Smart destinations". TourGUNE Jounal of tourism and human mobility, issue0, pág. 15-21.

Liburd, J. J.

2012. "Tourism research 2.0". Annals of Tourism Research, 39(2): 883-907.

Kim J, Tussyadiah IP.

2013. "Social networking and social support in tourism experience: the moderating role of online self-presentation strategies". Journal of Travel Tourism Marketing 30(1):78-92 
Mallor, E., González Gallarza, M., Fayos, T.

2013. "Qué es y cómo se mide el turismo cultural?. Un estudio longitudinal con series temporales para el caso español”. Pasos, 11(2): 269-284

McKercher, B \& Hilary du Cros

2002. "Cultural Tourism. The Partnership between tourism and cultural heritage management National Heritage Act”. Annals of Tourism Research, 30(2): 508-509

Mokre, M.

1998. "New technologies and established institutions" En: How Museum Present Themselves in the World Wide Web, Technisches Museum Wien, Austria.

O'Connor, P.

1999. Electronic information distribution in tourism and hospitality. Wallingford: CAB.

OMT

1995. "The state's role in protecting and promoting culture as a factor of tourism development and the proper use and exploration of the national cultural heritage of sites and monuments for tourism". Madrid.

Patuelli, Roberto; Mussoni, Maurizio; Candela, Guido

2013. "The effects of World Heritage Sites on domestic tourism: a spatial interaction model for Italy". Journal of Geographical Systems 15(3): 369-402.

Poon, A.

1993. Tourism, techonology and competitive strategies. Oxford: CAB International

Rasinger J, Fuchs M, Höpken W.

2007. "Information search with mobile tourist guides: a survey of usage intention". Information

Technologies and Tourism. 9(3-4):177-194

Richards, Greg.

2000. "Políticas y actuaciones en el campo del turismo cultural europeo", en El Turismo Cultural: el Patrimonio Histórico como fuente de riqueza, Fundación del Patrimonio Histórico de Castilla y León, Valladolid: 72.

Richards, Greg

2001. Cultural attractions and european tourism, Wallingford: CABI

Rypkema, D., Cheong, C., \& Mason, R.

2011. Measuring Economic Impacts of Historic Preservation.

Scali G., Segbert M., Morganti B.,

2002. "Multimedia applications for innovation in cultural heritage", En: Proceedings of 68th IFLA

Council and General Conference, August 2002, Glasgow, U.K., 2002.

Seaman, Bruce A.

2008. En Greg Richards (ed.): "Cultural Tourism: Global and Local Perspectives.” Journal of Cultural

Economics, September 2008, 32 (3): 231-236

Segittur

2013. Destinos turísticos inteligentes. URL: ttp://www.segittur.es/opencms/export/sites/segitur/.content/ galerias/descargas/documentos/Presentacin-Destinos-Tursticos-Inteligentes.pdf.

Serra Cantallops, A., \& Salvi, F.

2014. "New consumer behavior: A review of research on eWOM and hotels". International Journal of Hospitality Management, 36: 41-51.

Sheldon, P.

1997. Tourism information technologies. Oxford. CAB.

Suau, F.

2012. "El turista 2.0 como receptor de la promoción turística: estrategias lingüísticas e importancia de su estudio" Pasos, 10(4): 143-153

Stylianou-Lambert, T.

2010. "Gazing form home: Cultural tourism in Western Europe", Annals of Tourism Research, 16 (4): 477-491.

Styliani, S., Fotis, L., Kostas, K., \& Petros, P.

2009. "Virtual museums, a survey and some issues for consideration. Journal of Cultural Heritage", 10(4): 520-528.

Torres Bernier, E. (coord.)

2006. Estructura de mercados turísticos. Barcelona: UOC. 
Timothy, Dallen J.

2011. Cultural Heritage and Tourism: An Introduction. Channel View Publications

UNESCO

2014. Lista del Patrimonio Mundial en http://portal.unesco.org/es/ev.php-URL_ID=45692\&URL_DO=DO_

TOPIC\&URL_SECTION=201.html accedido 10/03/2014

Volo, S.

2010. "Bloggers' reported tourist experiences: Their utility as a tourism data source and their effect on prospective tourists". Journal of Vacation Marketing, 16(4): 297-311.

Wang D, Fesenmaier DR.

2013. "Transforming the travel experience: the use of smartphones for travel". En Cantoni L, Xiang $\mathrm{Z}$ (eds.) Information and communication technologies in tourism 2013. Springer, Vienna/New York Wang D, Park S, Fesenmaier DR

2011. "The role of smartphones in mediating the touristic experience". Journal of Travel Research 51(4):371-387

Zeng, B., \& Gerritsen, R.

2014. "What do we know about social media in tourism? A review". Tourism Management Perspectives, 10: $27-36$ 